\title{
La estructura de oportunidad política transnacional y el giro relacional en el análisis de la participación política y el asociacionismo migrante
}

\author{
Natalia Moraes Mena \\ Isabel Cutillas Fernández \\ Universidad de Murcia \\ nmoraes@um.es; isabelmaria.cutillas@um.es
}

\section{Resumen}

En los estudios sobre los determinantes de la participación política de los migrantes es frecuente encontrar análisis basados en el enfoque de la estructura de oportunidades políticas (EOP). Este enfoque surge a partir de los estudios sobre movimientos sociales y activismo cívico. Frente a los abordajes que explicaban la acción colectiva a partir de la agregación natural de intereses comunes, fruto de los procesos de interacción estratégica, de la movilización de recursos o del nivel socioeconómico de los actores, esta perspectiva entiende que la misma tiene más que ver con la existencia de un contexto político determinado que favorece el desarrollo de ciertas acciones e iniciativas cívico-políticas. El enfoque de la EOP es actualmente uno de los dominantes en el análisis del activismo migrante. El objetivo de este artículo es reflexionar sobre estos debates centrándonos en las principales limitaciones de la perspectiva de la EOP. Para ello, nos basaremos en el análisis del asociacionismo migrante en España y en los vínculos cívico-políticos de los migrantes con sus países de origen. Este trabajo pretende ofrecer un acercamiento teórico-analítico a partir de los resultados de las investigaciones que en los últimos años se han desarrollado en España sobre dicha temática. A partir de esta discusión, el artículo presenta una propuesta analítica relacional y dinámica de las principales dimensiones que entendemos deben tenerse en cuenta a la hora de abordar el asociacionismo y la participación cívico-política de los migrantes.

Palabras clave: migrantes; participación política; asociacionismo migrante; estructura de oportunidades políticas; perspectiva transnacional 


\begin{abstract}
The transnational political opportunity structure and the relational turn in the analysis of political involvement and the migrant associative movement
\end{abstract}

In migrant political participation studies it is common to find approaches based on the political opportunity structure (POS); a perspective that emerged from studies on social movements and civic activism. POS confronts other approaches that conceive collective action based on the natural aggregation of common interests. Instead of this, POS highlights the relevance of political context to explain the development of several political practices of migrants. POS is currently one of the dominant approaches in analyzing migrant activism. The aim of this article is to reflect on these debates and highlight the most important constraints of this perspective. To do so, we focus on immigrants' self-organizations in Spain and the links between them and their countries. The article presents a proposal of a relational and dynamic research framework in order to take into account the main factors that should be considered when dealing with migrants' civic and political participation.

Keywords: migrants; political participation; immigrant organizations; political opportunity structure; transnational perspective

\title{
Sumario
}

\section{Introducción}

2. Del enfoque de la estructura de oportunidades políticas al giro hacia

la relacionalidad

3. Limitaciones de la perspectiva de la EOP en los estudios sobre la organización y la movilización migrante
4. Los estudios sobre asociacionismo migrante en España y la perspectiva de la EOP

5. A modo de conclusión: una propuesta de marco analítico para el estudio del asociacionismo migrante

Referencias bibliográficas

\section{Introducción}

Los estudios sobre la participación política, la movilización y el asociacionismo migrante han centrado sus ejes de análisis fundamentalmente en tres cuestiones. En primer lugar, en la relación entre la organización étnica de cada uno de los grupos de migrantes y la participación política. En segundo lugar, en el análisis de la influencia de la estructura de oportunidades políticas (EOP) de la sociedad receptora sobre el asociacionismo y la participación política de los migrantes. Y, por último, sobre todo en los últimos quince años, ha cobrado relevancia el estudio de las prácticas transnacionales de los migrantes y los múltiples vínculos transnacionales que mantienen con diversos actores de los países de origen.

El primer grupo de estudios, vinculado al concepto de ethnic civic community (Fennema y Tillie, 2001), ha centrado su interés en analizar las diferencias en los niveles y en los espacios de participación política entre los distintos grupos de migrantes en una sociedad determinada, siendo objeto de análisis las redes sociales y el capital social étnico que poseen los diferentes grupos y cómo determinan las mismas su organización y su movilización. En este sentido, algunos de estos trabajos han tratado de mostrar la influencia de la organización étnica 
grupal en el conjunto de prácticas asociativas - no solo en las que tienen un componente étnico- $\mathrm{y}$ en la participación política individual de los migrantes (Jacobs y Tillie, 2004).

Por otro lado, los enfoques de la EOP han centrado su análisis en la influencia que, sobre el surgimiento, el desarrollo, la actuación y el empoderamiento de las asociaciones y de las organizaciones migrantes, ejercen los factores contextuales e institucionales de la sociedad de destino. Esta es actualmente una de las perspectivas más extendidas en el análisis del activismo migrante. Las investigaciones enmarcadas en esta línea han debatido acerca de cuál es la relevancia del Estado-nación en la definición del contexto de participación del tejido asociativo migrante. De este modo, algunos estudios defienden la relevancia del Estado (Koopmans, 2004), mientras que otras posiciones (Vermeulen, 2005; Soysal, 1994) advierten de la importancia de lo local y lo supranacional en la configuración de la estructura de oportunidades políticas.

Por su parte, el enfoque transnacional viene poniendo de relieve la vinculación de las asociaciones de migrantes con sus países de origen y cómo estos vínculos influyen en la configuración, la actividad y el desarrollo de las asociaciones (Schrover y Vermeulen, 2005; Portes et al., 2006), lo que supera la visión de la participación cívico-política de los migrantes como una realidad nacional, puesto que se entiende que es un fenómeno que condiciona y es condicionado por más de un contexto sociopolítico.

A pesar de que cada una de estas perspectivas ha contribuido a alcanzar un mayor conocimiento sobre las dinámicas asociativas de los migrantes y sus prácticas cívico-políticas, no logran, por sí solas, explicar los múltiples factores que condicionan sus prácticas organizativas. Si las oportunidades políticas son estructurales, externas y escapan al control de los agentes, ¿cómo influyen estos en las mismas? Si las estructuras de oportunidad determinan las prácticas, ¿cómo influyen las limitaciones? Si los migrantes mantienen vínculos transnacionales, ¿̨las estructuras de oportunidad no lo serán también? Si la capacidad organizativa depende de los recursos y del capital social de los migrantes, ¿cómo explicar los cambios que se registran a lo largo del tiempo en las asociaciones? Si las estructuras de oportunidad son las mismas para los diversos grupos de migrantes, : por qué unos se organizan y se movilizan y otros no, o no tanto? Para conocer por qué y cuándo surgen, desaparecen o cambian las organizaciones, cómo se organizan, qué acciones desarrollan y por qué y cuál es el impacto de su acción, debemos incorporar una perspectiva dinámica y relacional que incorpore diferentes dimensiones y que sea capaz de articular las propuestas de estas diferentes perspectivas. El objetivo del presente artículo es proponer un marco analítico relacional que nos permita comprender mejor estas cuestiones. Para ello, en este trabajo nos centramos, en primer lugar, en discutir el enfoque de las estructuras de oportunidades políticas aplicado inicialmente al estudio de los movimientos sociales. Analizamos las contribuciones más relevantes de esta perspectiva, así como sus principales limitaciones. En segundo lugar, a partir de lo que nos han permitido conocer las investigaciones desarrolladas sobre el asociacionismo y las prácticas cívico-políticas de los migrantes en España, 
mostramos por qué es relevante incorporar una perspectiva transnacional de la EOP en los estudios sobre la movilización migrante. En este análisis, prestamos especial atención al asociacionismo uruguayo, no desde la consideración de este como un ejemplo representativo del tejido asociativo migrante, sino como un caso específico de estudio que nos permite poner en evidencia las principales limitaciones de un enfoque de la EOP que no tiene en cuenta una perspectiva transnacional. Finalmente, proponemos un marco analítico multidimensional para estudiar esta temática.

\section{Del enfoque de la estructura de oportunidades políticas al giro hacia la relacionalidad}

El concepto estructura de oportunidades politicas es utilizado por primera vez por Eisinger (1973) al analizar las movilizaciones de protesta que tuvieron lugar en la década de 1960 en diferentes ciudades de Estados Unidos. Este autor mostraba cómo distintas estrategias y acciones del Estado podían dar lugar a estructuras abiertas o cerradas que influían en las movilizaciones. Como estructuras abiertas, mencionaba la forma de gobierno y el grado de respuesta que daba este a los diferentes grupos a través de las políticas llevadas a cabo. Dicha perspectiva comienza a adquirir relevancia como crítica a la falta de atención prestada por la teoría de la movilización de recursos hacia las dimensiones contextuales y estructurales para explicar el desarrollo de las asociaciones sociales. Las movilizaciones no se producían en el vacío, sino que estaban estructuradas y determinadas por las dinámicas del contexto político en el que tenían lugar. Fue sin duda Charles Tilly (1978) quien desarrolló con mayor profundidad este enfoque, que posteriormente se nutrió de las aportaciones de McAdam (1982) y Tarrow (1997). Para Tarrow (1997: 109): «[...] la acción colectiva prolifera cuando la gente tiene acceso a los recursos necesarios para escapar a su pasividad habitual y encuentra la oportunidad de usarlos». Algunos han visto a este enfoque como una nueva versión de la teoría de la movilización de recursos (Cohen y Arato, 2000), sin embargo, Tarrow (1997) entiende que no se pueden confundir ambas perspectivas, dado que el enfoque de la EOP no se centra en el análisis de la movilización de los recursos internos, sino en el de los recursos externos al grupo que alientan las prácticas organizativas y la movilización.

Además de los factores institucionales, la perspectiva de la EOP llama la atención sobre la importancia de la existencia de élites estatales o gubernamentales convertidas en aliados o en oponentes de los grupos y de los movimientos sociales. Estas élites y estos grupos con poder pueden dificultar o facilitar el acceso a las instituciones políticas. En este sentido, como señala Tarrow (1997), las élites pueden actuar como aliadas, ya sea por aspectos relacionados con su etnicidad, su género, sus orientaciones, sus intereses, sus pertenencias, etc. ${ }^{1}$.

1. Como ejemplo en este sentido, Eisinger (1973) llamaba la atención sobre el hecho de que el grado de presencia de representantes afroamericanos en las autoridades locales era un indicador del grado de mayor apertura institucional. 
Cuadro 1. Dimensiones de la estructura de oportunidades políticas

\begin{tabular}{|c|c|c|c|c|c|}
\hline $\begin{array}{l}\text { Espacio o canales } \\
\text { del sistema político }\end{array}$ & $\begin{array}{l}\text { Actores } \\
\text { principales }\end{array}$ & EOP como & Subdimensi & nes 0 aspectos & \\
\hline \multirow{3}{*}{$\begin{array}{l}\text { Estructura } \\
\text { y procesos } \\
\text { institucionales }\end{array}$} & \multirow[t]{3}{*}{ El Estado } & \multirow[t]{3}{*}{ Apertura } & $\begin{array}{l}\text { Estructura } \\
\text { de input }\end{array}$ & $\begin{array}{l}\text { Estructura formal del Estado: } \\
\text { federalismo, sistema electoral, etc. }\end{array}$ & $(+)$ \\
\hline & & & & $\begin{array}{l}\text { Estilo político. Prácticas } \\
\text { informales, agentes estatales } \\
\text { respecto a grupos y movimientos. }\end{array}$ & $\begin{array}{l}\text { E } \\
\text { S } \\
T \\
\text { A }\end{array}$ \\
\hline & & & $\begin{array}{l}\text { Estructura } \\
\text { de outputs }\end{array}$ & $\begin{array}{l}\text { Capacidad de la acción estatal para } \\
\text { el desarrollo de políticas. }\end{array}$ & B \\
\hline \multirow{2}{*}{$\begin{array}{l}\text { Élites y sistemas } \\
\text { de partidos }\end{array}$} & \multirow{2}{*}{$\begin{array}{l}\text { Élites y partidos } \\
\text { políticos }\end{array}$} & \multirow[t]{2}{*}{ Acceso } & \multicolumn{2}{|c|}{ «Influyentes» aliados u oponentes. } & i \\
\hline & & & \multicolumn{2}{|c|}{ Fragmentación y división entre élites. } & D \\
\hline \multirow[t]{2}{*}{ Sociedad civil } & \multirow{2}{*}{$\begin{array}{l}\text { Grupos y } \\
\text { movimientos, } \\
\text { opinión publica }\end{array}$} & \multirow[t]{2}{*}{ Apoyo } & \multirow{2}{*}{\multicolumn{2}{|c|}{ Grupos y movimientos aliados u oponentes. }} & $\begin{array}{l}A \\
D\end{array}$ \\
\hline & & & & & $(-)$ \\
\hline
\end{tabular}

Fuente: Navarro et al., 2006.

Estos aliados potenciales o influyentes pueden abrir las puertas del Estado o de los partidos políticos. Pero no solo las alianzas con élites o grupos de poder contribuyen a generar un contexto favorable para el desarrollo de determinadas movilizaciones. También los vínculos con otros movimientos o grupos, o la generación de una opinión pública favorable (o contraria) a las acciones del movimiento pueden influir en su desarrollo y alcance (Rucht, 1996).

A partir de los diferentes aportes de las teorías de las EOP, como vemos en el cuadro 1, Navarro et al. (2006) presentan una clasificación de las dimensiones de la misma, teniendo en cuenta tanto su grado de apertura como la existencia de actores que facilitan el acceso y que dan apoyo a los movimientos.

Si bien esta perspectiva - junto a las teorías de la movilización de recursos y al enfoque de marcos de acción colectiva - tuvo una gran repercusión en el estudio de los movimientos sociales hasta bien entrada la década de 1990, no ha estado exenta de críticas. Se ha cuestionado, en primer lugar, la invisibilidad de las potencialidades de influencia de los movimientos en el cambio de las estructuras políticas. El origen, la dinámica, las formas y las consecuencias de la acción de los movimientos sociales son supeditados a la apertura o al cierre de la estructura de oportunidades políticas, dejando de lado la posibilidad de agencia y de que los movimientos puedan influir a su vez en la transformación de estas estructuras de oportunidad (Delgado, 2007). Como han señalado Gamson y Meyer (1999: 403): «Las oportunidades catalizan la acción política, pero los movimientos también consiguen ampliar el espectro de las oportunidades».

Por otro lado, al explicar la movilización a partir de factores externos a los grupos, se pierde de vista la diversidad intrínseca de las organizaciones y de los movimientos, por lo que no todos ellos estarán en igualdad de 
condiciones a la hora de identificar una apertura institucional. El enfoque no logra responder por qué, en ocasiones, algunos grupos aprovechan la oportunidad política y otros no. Además, la oportunidad no existe hasta que no se percibe como tal. Es decir, no basta con que haya una apertura para la movilización, sino que es necesario que esta sea percibida como oportunidad por los sujetos que se ponen en marcha. Al mismo tiempo, no es probable que se aproveche una oportunidad si no existe una infraestructura organizativa capaz de canalizar los procesos de movilización (McAdam et al., 1999). Este enfoque, además, no tiene en cuenta el componente simbólico-expresivo de la acción, puesto que deja de lado la importancia de la producción de significados o lo que se ha llamado política de la significación (Benford y Snow, 2000).

Las limitaciones de perspectiva de la EOP están relacionadas también con su refutación empírica. Atendiendo a su esquema de interpretación, se presupondría que en un sistema represivo habría mayores restricciones para la acción colectiva, sin embargo, la represión latente o efectiva ha redundado en ocasiones en un incremento de la oportunidad política. Véase, por ejemplo, en el caso de América Latina, los estudios de Quiroga (2009) sobre Bolivia y los de Markarian (2006) sobre las redes transnacionales de defensa de los derechos humanos en las dictaduras del Cono Sur. La cuestión central sobre las limitaciones de esta perspectiva tiene que ver, fundamentalmente, con el hecho de si se puede considerar a la estructura de oportunidad política como una variable analítica independiente o si se la debe considerar como un factor más de una amalgama formada por un conjunto de factores (Bousetta, 2000). Como señala Delgado (2007), el «giro hacia la relacionalidad» de algunos de los autores más destacados del enfoque de la EOP podría interpretarse como una estrategia para superar estas limitaciones. Para este autor, Dynamics of Contention, de McAdam et al. (2001), es un claro compromiso con una mirada más relacional y dinámica de los factores que influyen en los movimientos sociales $y$, a pesar de las críticas que su propuesta ha recibido, contribuye sin duda a realizar una interpretación más compleja de la acción colectiva. McAdam et al. (2001) plantean un modelo dinámico de movilización en el que se tienen en cuenta los aspectos siguientes:

1) No solo las estructuras de oportunidad, sino también las amenazas al estudiar el contexto. Considerando, además, las oportunidades y las amenazas como elementos construidos e interpretados durante la interacción.

2) La apropiación social, esto es, la capacidad y los esfuerzos de los sujetos que están detrás de las estructuras de movilización.

3) La construcción social, dando primacía a elementos y a procesos interpretativos para explicar las trayectorias de la acción colectiva.

Por otro lado, se ha llamado la atención también en la dimensión discursiva de la EOP que moldea la identificación colectiva de las minorías étnicas (Statham, 1999). 


\section{Limitaciones de la perspectiva de la EOP en los estudios sobre la organización y la movilización migrante}

La perspectiva de la EOP, desarrollada fundamentalmente para explicar las prácticas de los movimientos sociales, pasó luego a ser incorporada al análisis del movimiento migrante y étnico. Uno de los primeros autores que utilizó este enfoque para el análisis de las migraciones fue Ireland (1994) en su estudio sobre el caso de Francia y Suiza. Para este autor, las movilizaciones de los migrantes no se podían explicar únicamente atendiendo a factores como la clase social y la etnicidad, dimensiones principales tenidas en cuenta hasta el momento en el análisis del asociacionismo inmigrante, sino que, por el contrario, debían tenerse en cuenta los contextos políticos e institucionales específicos en los que las prácticas colectivas de los migrantes tenían lugar. A partir de su trabajo, un gran número de investigadores ha analizado las diferencias locales que configuran las distintas estructuras de oportunidades políticas (Vermeulen, 2005), con lo que actualmente esta perspectiva se ha convertido en una de las principales en el análisis del activismo migrante ${ }^{2}$.

La enorme importancia que la perspectiva de la EOP ha tenido en el análisis del activismo migrante no ha evitado, sin embargo, que esta haya sido objeto de críticas y de revisiones. Algunos de los cuestionamientos que ya recibía el enfoque en su análisis de los movimientos sociales pueden aplicarse también a los estudios sobre la movilización y la organización migrante. La crítica de Melucci (2002) sobre el estudio de los movimientos sociales es válida también para el análisis del asociacionismo migrante: no podemos comprenderlo porque solo miramos la punta del iceberg, nos centramos en lo visible, dejando de lado o no teniendo en cuenta lo latente. Lo que no buscamos ver, creemos que no existe (Moraes et al., 2013).

Se ha criticado que su carácter excesivamente institucionalista deja de lado otras dimensiones relevantes. Bousetta (2000) ha llamado la atención sobre algunas de sus principales limitaciones, entre ellas: el no considerar la influencia de los procesos no institucionales o de los aspectos vinculados a la infrapolítica, el marginar las variables relacionadas con la evolución socioeconómica y política del contexto receptor y las características de los diferentes grupos de migrantes, la organización interna de los distintos colectivos y las diferencias internacionales e intranacionales y étnicas, la concepción de los migrantes como agentes pasivos cuyas acciones están determinadas exclusivamente por la estructura política de oportunidad, la falta de atención a la dimensión simbólica de la movilización étnica y, en definitiva, la falta de articulación de los factores macro, meso y micro. Es necesario, desde su perspectiva, un marco analítico que tenga en cuenta el rol de las minorías étnicas como actores políticos, el contexto en el que estos actúan y la posición que ocupan en el mismo.

2. Un análisis del activismo y del asociacionismo migrante desde la perspectiva de la EOP puede encontrarse en el número 31 del Journal of Ethnic and Migration Studies, que en 2005 dedica un monográfico especial a las organizaciones de migrantes. 
En la misma línea, en su estudio sobre la acción colectiva de los migrantes latinos en Londres, Però (2009) muestra que la EOP puede explicar parte de la movilización y de la organización de los migrantes. «El contexto multicultural británico, organizado alrededor de comunidades étnicas más antiguas, ha contribuido a que los migrantes latinoamericanos de diferentes nacionalidades se movilicen alrededor de una identidad étnica común en el Frente Latino» (Però, 2009: 177). Sin embargo, también presenta algunas limitaciones. En primer lugar, este autor critica la existencia de lo que llama una cierta «tendencia etnicista» en los estudios basados en la EOP, ya que, si bien tienen en cuenta las variaciones de las movilizaciones entre diversos grupos étnicos en un mismo contexto específico, no suelen explicar los cambios que se pueden registrar dentro de un mismo grupo. Es decir, se concibe de esta forma a los grupos étnicos como grupos homogéneos sin diversidades internas, con las mismas orientaciones y prácticas influidas fundamentalmente por su contexto. Otra de las críticas que realiza Però está relacionada con el hecho de que, generalmente, desde esta perspectiva, se tiene una visión estrecha de lo que incluye la EOP, es decir, generalmente solo se incluye el aparato político e institucional, dejando de lado, como mostrábamos en el cuadro 1, los actores que ayudan a acceder a los movimientos o que les sirven de apoyo, como por ejemplo, otras organizaciones de la sociedad civil, los medios de comunicación y las actitudes y/o la opinión pública que existe en relación con los distintos colectivos de migrantes. Al poner demasiado énfasis en el entorno institucional, se presenta a los migrantes como agentes pasivos cuyas acciones están estructuralmente determinadas por los factores institucionales (Però y Solomos, 2010: 9). Los factores que, según Però (2009), pueden explicar la movilización de los migrantes son: la socialización, la historia, la experiencia y los valores políticos de los migrantes y las condiciones de vida en el país de recepción. Desde su perspectiva, las «limitaciones estructurales de oportunidades» pueden ser más movilizadoras que las oportunidades políticas. Por último, otro elemento a tener en cuenta es el relacionado con las redes y el capital social de los migrantes. A través de las redes establecidas, los migrantes han hallado la posibilidad de organizarse y de movilizarse.

Además, no basta solo con analizar la estructura de oportunidad y la apertura que favorece la movilización migrante, sino que también se deberá considerar si estas oportunidades son percibidas y cómo son percibidas. Atendiendo a la dimensión discursiva de la EOP, será necesario observar también cómo se construyen determinadas estructuras de sentido que favorecen la movilización y la organización de determinados grupos de migrantes. La condición inmigrante (Pedreño, 2005) se articula con otros ejes de autoadscripción y adscripción externa - como clase, etnia, raza, género, entre otros- que será necesario tener en cuenta a la hora de analizar la organización colectiva, la movilización y las dinámicas asociativas en contextos locales específicos. Los procesos de homogeneización interna y diferenciación externa, que tienen lugar a partir de las características de los contextos nacionales y locales de recepción, están relacionados con los procesos de construcción de sentido en términos de la 
posición social que los diferentes grupos de migrantes ocupan. A su vez, este proceso tendrá que ver también con las jerarquías de nacionalidades estructuradas en dicho contexto (Moraes, 2010), determinadas tanto por el marco jurídico que define la nacionalidad y la ciudadanía (quién puede o no y qué debe hacer para obtenerla), como por las representaciones que la sociedad de acogida tiene de los diferentes grupos nacionales de migrantes.

Además de los factores explicativos vinculados al país de recepción, el enfoque transnacional ha puesto en evidencia la necesidad de adoptar una perspectiva más amplia de la estructura de oportunidad política en el análisis de la movilización y las prácticas cívico-políticas de los migrantes. Esta quizás es una de las principales críticas que se puede hacer al enfoque de la EOP en el contexto global y transnacional actual. Las prácticas políticas de los migrantes no deben analizarse solo desde el país de residencia de estos, sino que los estudios deben situarse también en el contexto político institucional de origen y destino (Østergaard-Nielsen, 2009). Diversos estudios han puesto de relieve el papel de las iniciativas de vinculación de los estados de origen en las prácticas políticas de los migrantes (Østergaard-Nielsen, 2003; Moraes et al., 2009). Aunque, pese a ello, como también se ha señalado, estas estrategias de vinculación pueden adolecer de credibilidad y no llegar efectivamente a todos los migrantes.

Por otro lado, los Estados de origen no son los únicos actores que configuran las EOP desde la perspectiva transnacional. Pueden desempeñar un papel importante también los partidos políticos y otras organizaciones civiles del país de origen. En el enfoque transnacional de las EOP no puede quedar fuera tampoco el Estado receptor, que «[...] juega un papel central al marcar los límites de la inclusión, exclusión, ciudadanía, permitiendo o prohibiendo las distintas formas de movilización política dentro de sus fronteras» (Østergaard-Nielsen, 2009: 27). Varios estudios han mostrado cómo la EOP del país de recepción influye en la participación política en el país de origen, pero, al mismo tiempo, diferentes modelos de incorporación política llevados a cabo por diferentes Estados de recepción pueden fomentar prácticas políticas transnacionales similares (Østergaard-Nielsen, 2003). Aspectos todos ellos que tornan compleja y multidimensional la interpretación de los determinantes de la movilización y de la organización local, nacional y transnacional de los migrantes. La aplicación del enfoque de EOP desde una perspectiva transnacional tampoco debe olvidar la diversidad existente dentro de los mismos colectivos de migrantes. «Los distintos grupos de migrantes dentro de un mismo país receptor pueden tener acceso diferente y elegir estrategias muy distintas» (Østergaard-Nielsen, 2009: 29). Además, es importante tener en cuenta la importancia de la cultura política del país de origen y de la cultura política y el aprendizaje político que los propios migrantes han configurado en ambos países, así como también el contexto histórico-político premigratorio (Moya, 2005). Las prácticas cívicopolíticas de los migrantes y sus organizaciones son parte de un mecanismo continuo de retroalimentación, a través del cual los migrantes influyen y son influenciados por el ambiente político e institucional local, nacional y transnacional (Østergaard-Nielsen, 2009). 


\section{Los estudios sobre asociacionismo migrante en España y la perspectiva de la EOP}

Desde la década de 1990, en la que surgen los primeros estudios e investigaciones acerca del asociacionismo inmigrante en España, el interés que este ámbito ha despertado en académicos e investigadores ha sido creciente. En estos estudios pueden distinguirse dos etapas, «ambas relacionadas con el modo de mirarse en ellas al tejido asociativo constituido por las asociaciones» (Aparicio, 2011:3). Mientras que en un primer periodo se estudia la relación entre el asociacionismo de los migrantes y su integración social (fundamentalmente a través de aspectos como el número de asociaciones, los objetivos, las actividades que realizan, etc.), posteriormente los estudios centran la atención en la participación de las asociaciones en el ámbito público y su papel en la integración política de los migrantes (Aparicio, 2011).

Muchas de estas investigaciones han sido muy críticas con el impacto real del asociacionismo y su escasa contribución en la integración cívica y política de los inmigrantes en España. Por un lado, se ha señalado la dificultad de estas asociaciones para ser consideradas como actores políticos representantes de los intereses de los migrantes, mostrando las escasas relaciones y contactos que se producen entre las asociaciones de migrantes y los agentes públicos y políticos (Morales y González, 2006), debido tanto a la relación clientelar que en ocasiones se produce con la administración (Veredas, 2003; Martín Pérez, 2004), como a su problemática integración vertical (con las autoridades), horizontal (con otra organizaciones) e interna (Toral, 2011). Aboussi et al. (2013) analizaron, para el contexto andaluz, la situación de estos tres espacios de conflicto para el asociacionismo inmigrante, considerándolos ámbitos de análisis cruciales en el estudio de la EOP real del tejido asociativo. En relación a la vinculación que las asociaciones de migrantes mantienen con la administración, algunos autores han apuntado también a la influencia que la misma, en tanto que requeridora de un interlocutor reconocido, ha ejercido en la creación, desarrollo o actividad de estas asociaciones (Garreta, 2011; Lacomba et al., 2015), y han llamado la atención sobre la reducción de su capacidad reivindicativa y de movilización tanto por la dependencia económica de las ayudas públicas, como por la cooptación de sus líderes (Toral, 2011). En este sentido, Mata ha mostrado cómo se promueve por parte de la administración un tejido asociativo dependiente a través de las subvenciones, la financiación y ayuda (Mata, 2011). Sin embargo, otros estudios han apuntado también cómo algunas organizaciones han desafiado los espacios institucionales de participación promovidos por la administración (Moncusí y Escala, 2013). Por otro lado, se ha señalado que las asociaciones no han sido determinantes en la integración política de los migrantes por su escasa representatividad, el papel desarrollado por sus líderes y las actividades llevadas a cabo (Aparicio, 2011), así como por la prevalencia de las iniciativas orientadas a la promoción cultural o al reforzamiento étnico (Aparicio y Tornos, 2005).

Una parte importante de los más recientes análisis sobre la organización y la movilización migrante se ha basado en la perspectiva de la EOP. Se ha señalado 
que la EOP (legal, institucional y discursiva) es uno de los factores clave a la hora de entender el grado de participación cívica y política de los inmigrantes (Morales et al., 2005). González et al. (2009), en el estudio desarrollado en Barcelona, Madrid y Murcia sobre el impacto que las políticas de participación ciudadana y de incorporación de los inmigrantes tienen sobre la estructura y la orientación del asociacionismo migrante, señalan que la EOP afecta al tamaño y a la consolidación del tejido asociativo inmigrante, así como a las actividades que desarrollan. También Toral ha señalado que la EOP influye en el modo cómo los actores se definen a sí mismos y cómo se sitúan en el campo de juego (Toral, 2010). Basándose en la propuesta analítica de Soysal (1994) sobre las tipologías de los regímenes de incorporación, y tomándolos como tipos ideales de la EOP, Goñalons (2007) analiza qué estructura de oportunidad ofrece el Estado español para la incorporación de los inmigrantes. En su análisis muestra cómo el modelo español resulta desfavorable para la participación de las asociaciones de inmigrantes en las instituciones públicas, ya que se permite la emergencia de tales movimientos, pero no se les considera actores legítimos para la participación en el ámbito público (Goñalons, 2007).

La contribución de estos estudios al campo de análisis del asociacionismo y la participación cívico-política de los migrantes ha sido relevante, dado que nos han permitido conocer algunos de los factores determinantes de la organización y de la movilización cívico-política de los inmigrantes en España, mostrando la influencia de la estructura de oportunidad política en sus prácticas individuales y colectivas. Sin embargo, el hecho de que buena parte de estos trabajos se hayan centrado exclusivamente en la EOP del contexto de recepción hace que su explicación sobre la acción colectiva de los migrantes sea incompleta o nos permita entender solo en parte la misma. Si no adoptamos un enfoque transnacional y una perspectiva multidimensional y relacional que vaya más allá de la EOP, nuestras interpretaciones pueden ser limitadas y podemos dejar de conocer parte de las razones y de las motivaciones de los cambios y de las actuaciones de las organizaciones de migrantes. Como mencionábamos antes, como no las buscamos, creemos que no existen. Adoptar este enfoque no significa considerar que todas las organizaciones de migrantes desarrollan prácticas transnacionales, sino que implica tener en cuenta que las acciones de los Estados de origen, así como las de otras instituciones y organizaciones de los países de origen, pueden influir en la organización de los migrantes en España y en sus prácticas cívico-políticas y viceversa. Esta perspectiva implica considerar además el nivel local-transnacional en el análisis de los vínculos y de las influencias entre los países de origen y de destino de los migrantes.

En esta línea, Cortés y San Martín (2010), en su estudio sobre el asociacionismo ecuatoriano y colombiano en España, han mostrado cómo la apertura de oportunidades políticas a nivel local y transnacional ha tenido un impacto importante en la intensidad y en las características de las acciones desarrolladas por las organizaciones de migrantes. Gadea y Albert (2011) han mostrado cómo el proyecto de codesarrollo Cañar-Murcia, que conectaba la Región de Murcia con la de Cañar en Ecuador, ha influido en la configuración del 
asociacionismo de los migrantes cañaris residentes en la Región, lo que ha propiciado su institucionalización formal, ha generado conflictos de liderazgo y de representación y ha transformado sus prácticas.

Las investigaciones que hemos desarrollado en los últimos años sobre los migrantes latinoamericanos en España nos han permitido mostrar cómo los Estados y otras instituciones del país de origen son actores que desempeñan un papel importante tanto en la creación como en la dinamización e incluso en la fractura de las asociaciones de migrantes en España (Moraes et al., 2009; Moraes et al., 2013). El análisis de las transformaciones del asociacionismo uruguayo en España nos ofrece, como veremos, un claro ejemplo de la pertinencia del enfoque transnacional de la EOP. La organización de migrantes uruguayos en España adquiere un notable dinamismo entre los años 2002 y 2008 . Un período relevante, no solo porque es cuando se produce un aumento del número de inmigrantes uruguayos fruto de la crisis económica registrada en el país de origen, sino también porque en esos años se producen importantes cambios políticos en Uruguay que trajeron consigo una transformación sustancial de las políticas migratorias desarrolladas hasta ese momento en el país.

En ese período surgieron más de cuarenta asociaciones de uruguayos repartidas por las diversas comunidades autónomas de España. Las principales movilizaciones de estas asociaciones estuvieron relacionadas con lo que ØstergaardNielsen (2009) llama immigrant politics y emigrant politics ${ }^{3}$. En primer lugar, las movilizaciones estuvieron vinculadas con la defensa de los emigrantes en España y el cumplimiento del Tratado de 1870 firmado entre España y Uruguay, por el cual los uruguayos consideraban que tenían derecho a la residencia legal en España; en segundo lugar, por los derechos de los emigrantes en el país de origen, especialmente en la reivindicación del voto desde el exterior. Ambos tipos de movilizaciones se pueden denominar transnacionales, ya que se vieron influidas por acontecimientos en los dos países y porque en las mismas han intervenido actores sociales y políticos, tanto de España como de Uruguay. Pero no solo el Estado de origen, el de recepción y las condiciones específicas de incorporación local-transnacional han influido en este caso en la dinamización y en la transformación de las asociaciones de migrantes uruguayos. Las organizaciones de la sociedad civil creadas para defender los derechos de los emigrantes han tenido un importante papel como actores de apoyo. Entre ellos podemos destacar el de la Red Diáspora, creada en Uruguay, compuesta por un conjunto de organizaciones de la sociedad civil que trabajaban sobre la temática emigratoria. Las movilizaciones de esta red representaron un apoyo para las asociaciones de uruguayos en España, tanto en lo relativo a la defensa de sus derechos como inmigrantes en España, como a lo relacionado con sus derechos

3. El primer concepto hace referencia a las actividades políticas que llevan a cabo los migrantes para mejorar su situación en el país de acogida, y el segundo a aquellas que tratan de institucionalizar el estatus transnacional de los migrantes que siguen vinculados económica, social y políticamente al país de origen (Østergaard-Nielsen, 2009). 
políticos en el país de origen. La Red Diáspora se movilizó y desarrolló diversas estrategias de lobby ante el Gobierno uruguayo y ante los partidos políticos de dicho país en defensa de los derechos de los emigrantes. Gran parte de esta presión estuvo relacionada con conseguir que el Gobierno uruguayo y el Parlamento de dicho país se pronunciasen a favor del Tratado de 1870 y defendiesen la situación de los uruguayos que se encontraban sin residencia legal en España. Entre las principales acciones de presión hacia estos dos actores se puede señalar las reuniones mantenidas con el Gobierno y con los integrantes de la Comisión de Asuntos Internacionales del Parlamento uruguayo. Estos encuentros fueron decisivos para conseguir el apoyo de la Cámara de Representantes de Uruguay a través de una carta declaración al Parlamento español, en la que se defendía la vigencia de este tratado. La Red Diáspora también se movilizó en apoyo a las estrategias que habían promovido las organizaciones de migrantes uruguayos en España. Comenzó a recolectar firmas entre los españoles residentes en el país en apoyo a este tratado y promovió una recogida de firmas en Uruguay para ser entregadas al Gobierno español. El objetivo de esta acción era reforzar las iniciativas del Gobierno y del Parlamento uruguayos y, a través de este acto simbólico, mostrar el compromiso de los ciudadanos uruguayos y de los inmigrantes españoles en Uruguay con la causa.

La Red Diáspora logró introducir también la temática del Tratado de 1870 en la campaña electoral que se desarrollaba en Uruguay con motivo de las elecciones gallegas del año 2005. El BNG fue el partido que incorporó esta cuestión con mayor intensidad en la campaña realizada en el país. La Red Diáspora también tuvo un papel importante en la defensa del voto de los uruguayos desde el exterior, puesto que trabajó conjuntamente con los consejos consultivos y participó en la creación de la comisión por el voto de los uruguayos en el exterior. De esta forma, las organizaciones de migrantes uruguayos en España pudieron introducir el debate sobre sus derechos como inmigrantes y emigrantes en la esfera pública uruguaya y en la agenda política (uruguaya y española) a partir del apoyo y del lobby desarrollado por la Red Diáspora. Al mismo tiempo, las organizaciones de uruguayos en España se vieron influidas por las transformaciones sociales y políticas registradas en Uruguay. Las políticas de vinculación que se comenzaron a promover con el primer gobierno de Tabaré Vázquez generaron importantes cambios en la organización, en la gestión y en la dinámica asociativa. La creación de los consejos consultivos generó importantes tensiones en las asociaciones, lo que provocó cambios internos en algunas de las mismas, así como una redefinición de las relaciones y de las prácticas que venían desarrollando, tanto en España como hacia el país de origen. La investigación etnográfica transnacional desarrollada sobre el asociacionismo uruguayo puso en evidencia cómo las transformaciones de las asociaciones, así como las prácticas cívico-políticas de sus integrantes, no podían explicarse exclusivamente atendiendo a la apertura institucional en el país de residencia, sino que fundamentalmente estaban definidas por una mayor apertura de la estructura de oportunidad en origen y por el papel desarrollado por élites políticas y partidos políticos (facilitando acceso) y otras organizaciones de la 
sociedad civil, como la Red Diáspora (generando apoyo y repercusión pública) a las movilizaciones y reclamos de los migrantes en España.

Otro caso que muestra la importancia de factores transnacionales en la trasformación del movimiento asociativo son los cambios registrados en las federaciones de peruanos en España. El terremoto en 2007 en Perú propició el establecimiento de redes de ayuda y cooperación que se consolidaron en federaciones específicas como la FEDAP y la FEPERCAT. Ambas organizaciones recibieron el apoyo del Partido Socialista en el Gobierno catalán en el período y del APRA del Perú. Sin embargo, con el Gobierno de CIU, en 2010 se propició el surgimiento de una nueva asociación vinculada estrechamente a la democracia cristiana y a partidos conservadores peruanos (Moraes et al., 2013).

\section{A modo de conclusión: una propuesta de marco analítico para el estudio del asociacionismo migrante}

Las investigaciones sobre las organizaciones de migrantes desarrolladas en España que han adoptado un enfoque transnacional han puesto en evidencia la relevancia de un enfoque internacional de las oportunidades políticas. Estos estudios han mostrado cómo las dinámicas asociativas y la participación cívicopolítica de los migrantes se han visto influidas por aperturas institucionales, apoyos o limitaciones acontecidas tanto en los contextos de origen como en el territorio español. Estos estudios han mostrado también la necesidad de incorporar una perspectiva que articule diferentes escalas (local, nacional y supranacional) y a distintos actores (Estado, élites y partidos, organizaciones de la sociedad civil, etc.).

Si bien este enfoque de la EOP ayuda a comprender mejor el desarrollo de las organizaciones de migrantes, sus luchas y sus cambios a lo largo del tiempo, no es suficiente para lograr respuestas más comprensivas y complejas sobre cuándo, por qué y cómo se organizan los migrantes y crean asociaciones que los vinculan en tanto que inmigrantes, qué prácticas cívico-políticas llevan a cabo y cómo las mismas van cambiando a lo largo del tiempo. Para ello es necesario realizar un enfoque relacional y multidimensional que incorpore una perspectiva macro, meso y micro.

El marco de referencia analítico que proponemos busca incorporar tanto una visión amplia de la estructura de oportunidad política y sus limitaciones, como otros factores vinculados a las dinámicas organizativas internas, la posición social y simbólica de los diversos grupos de migrantes, el capital social que poseen sus integrantes y aspectos que tienen que ver con la infrapolítica y con las características individuales de sus integrantes y su proceso migratorio. $\mathrm{Al}$ mismo tiempo, buscar atender también al contexto socioeconómico y político del país de origen y de residencia e incluir una perspectiva histórica de los vínculos y de las relaciones entre actores de ambos contextos que podrían influir promoviendo o restringiendo las movilizaciones y las prácticas cívico-políticas. En la figura 1 se esquematiza esta propuesta. 
Figura 1. Marco analítico para el estudio del asociacionismo y la participación cívico-política migrante

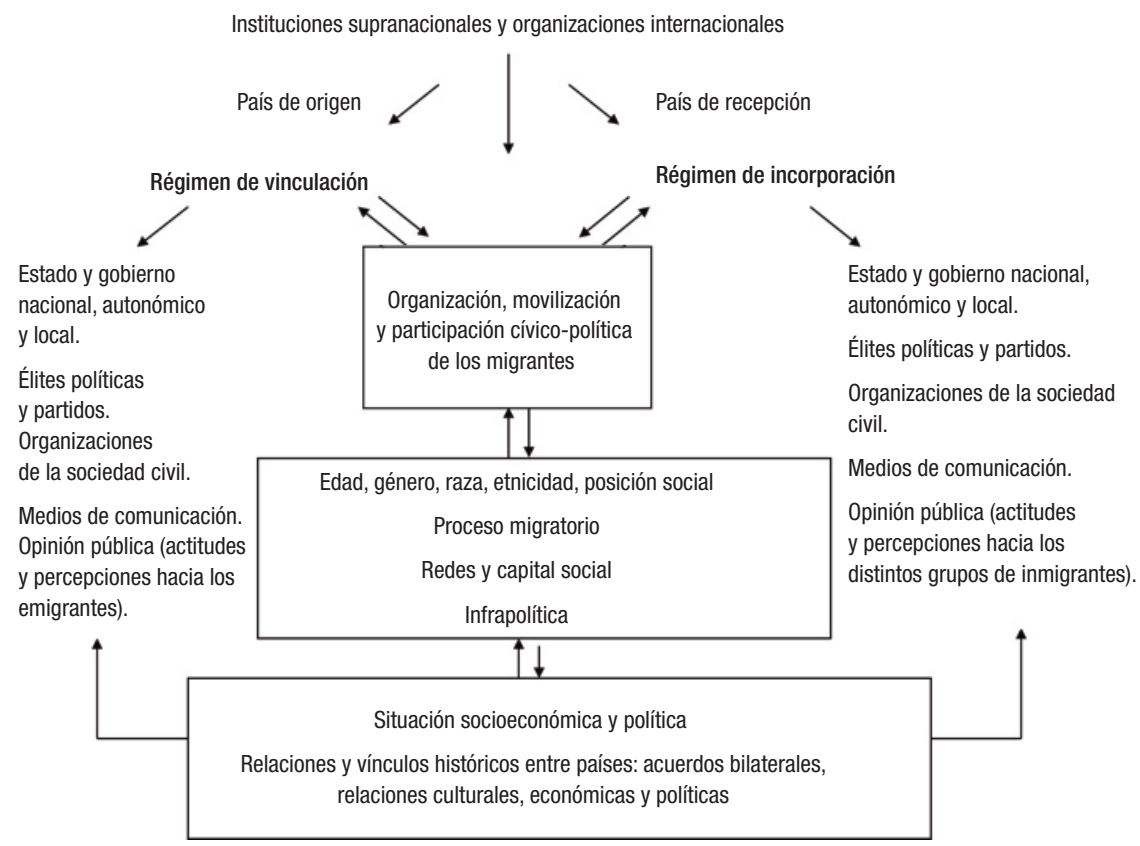

Fuente: elaboración propia.

El régimen de vinculación del país de origen y el régimen de incorporación del país receptor constituyen estructuras de oportunidad o de limitaciones o amenazas para la creación y el desarrollo de las organizaciones de migrantes e inciden en la movilización y en la participación cívico-política de los mismos. A su vez, estas estructuras que definen la apertura, el acceso y el apoyo a las organizaciones de migrantes y sus luchas están influidas por instituciones supranacionales y organizaciones internacionales que pueden instar a realizar cambios en las características de los diferentes regímenes de vinculación y de incorporación, promoviendo, por ejemplo, la aplicación de directrices para el control de las fronteras y la gestión de la inmigración o denunciando la violación de los derechos humanos de los inmigrantes.

Pero la influencia de la EOP transnacional no explica por sí sola la movilización ni el asociacionismo migrante. Los migrantes, como sujetos políticos activos y con voz, pueden interpretar de diversa manera esas oportunidades y amenazas y actuar en consecuencia. La capacidad para aprovechar o no las mismas depende de diversos factores individuales y colectivos (las características sociodemográficas y socioeconómicas de los migrantes, su capital cultural y político, las redes y el capital social que poseen sus organizaciones, su proceso migratorio y la posición que ocupan en tanto que inmigrantes y en tanto que 
nacionales de un determinado país o miembros a su vez de un determinado grupo étnico o religioso, como sujeto «otro» o como alteridad en la sociedad de origen y en la de recepción. Dado que la identificación colectiva de los migrantes y de las minorías étnicas se configura no solo por las prácticas y por las estructuras que definen su posición social y que determinan su «condición inmigrante y emigrante», sino también por las representaciones que, sobre ellos, en tanto que grupo, son construidas en la sociedad de origen y de destino. Esa construcción del otro, por ejemplo, como «gusano», «traidor» o «paisano» (hacia el emigrante) o de "moro», «sudaca» o «latino» (hacia el inmigrante), también debe tenerse en cuenta a la hora de analizar la movilización y la organización colectiva de los migrantes, y muestra la necesidad de incorporar en el análisis los discursos, las percepciones y las representaciones locales y nacionales sobre la inmigración y la emigración. Estos marcos de significación y de construcción de la otredad influyen en los procesos de autoadscripción y adscripción externa de los grupos de migrantes e inciden también en los procesos colectivos de interpretación sobre las oportunidades y las limitaciones para la organización y la movilización.

Además de las características individuales y colectivas, se deben tener en cuenta también aspectos que tienen que ver con la infrapolítica, los cuales a menudo son dejados de lado en los análisis de la participación cívico-política de los migrantes. Nos referimos a ese «tercer espacio» (Scott, 2003) donde prácticas no específicamente políticas se convierten en espacios de resistencia que pueden contribuir a la emergencia de prácticas cívico-políticas definidas. No solo porque diversas prácticas desarrolladas por los migrantes, como fiestas, ritos, cuentos, canciones, juegos, que toman forma también a través de Internet se pueden convertir en espacios de resistencia y germen de acciones colectivas en el espacio público, sino también porque, como ha mostrado Bolzman (1997), la movilización cultural puede permitir acumular recursos que luego son utilizados más políticamente.

Finalmente, este modelo analítico propone incorporar una perspectiva histórica que permita tener en cuenta la influencia de los cambios en el contexto socioeconómico y político del país de origen y de recepción, y los vínculos sociales, culturales y políticos entre los Estados de referencia para los migrantes. No solo porque el contexto socioeconómico y político premigratorio, el contexto socioeconómico y político de recepción y las relaciones bilaterales entre los Estados pueden ayudarnos a entender el porqué de determinadas luchas asociativas, sino también porque nos pueden ayudar a comprender cómo se ha configurado la estructura política de oportunidad transnacional.

La propuesta que hemos presentado aquí no busca ofrecer un modelo cerrado de análisis, sino que más bien pretende ser un punto de partida para una interpretación más compleja, dinámica y relacional de la acción colectiva de los migrantes. Un marco interpretativo que desplace la mirada, en primer lugar, de las estructuras de oportunidad política del país de recepción a una concepción transnacional de las EOP en donde se tengan en cuenta tanto las oportunidades como las amenazas y tanto a los Estados como a los actores que ayudan al acceso 
y sirven de apoyo a las organizaciones de migrantes. Un desplazamiento también de lo estrictamente institucional a lo discursivo, que incorpore los marcos de significación a través de los cuales los grupos de inmigrantes se definen tanto en forma individual como colectiva. En segundo lugar, un desplazamiento de estas estructuras hacia la capacidad de agencia de los migrantes y hacia su capacidad de incidir y transformar estas estructuras al mismo tiempo. En tercer lugar, girar la mirada desde lo estrictamente considerado como cívico-político hacia otros espacios de construcción de resistencias y formas indirectas de expresión colectiva, como prácticas culturales y religiosas, desde donde se pueden articular diversas acciones que pueden influir en las dinámicas organizativas y en las luchas asociativas. Por último, este modelo interpretativo sugiere la necesidad de incorporar una perspectiva histórica de los contextos, de las relaciones y de los vínculos entre los mismos, así como de las transformaciones del campo migratorio y del movimiento asociativo de migrantes que nos ayude a situar las prácticas individuales y colectivas en un marco más global y relacional.

\section{Referencias bibliográficas}

ABoussi, M.; RAYA, E. y ESPADAS, M.A. (2013). «Asociacionismo inmigrante, tercer sector y administración pública en Andalucía: Dimensiones de política, necesidades de gobernanza y oportunidades para la ciudadanía activa». En: RAYA, E.; ESPADAS, M.A. y ABOUssI, M. (coords.). Inmigración y ciudadanía activa: Contribuciones sobre gobernanza participativa e inclusión social. Barcelona: Icaria.

APARICIO, R. (2011). «Participación ciudadana y asociaciones de inmigrantes». Oñati Socio-Legal Series, 1(3).

APARICIO, R. y TORNOS, A. (2005). Las redes sociales de los inmigrantes en España. Madrid: Ministerio de Trabajo y Asuntos Sociales. Documentos del Observatorio Permanente de la Inmigración.

BENFORD, R. y SNOw, D. (2000). «Framing processes and social movements: An Overview and assessment». Annual Review of Sociology, 26. <https://doi.org/10.1146/annurev.soc.26.1.611>

BOUSETTA, H. (2000). «Institutional theories of immigrant ethnic mobilization: Relevance and limitations". Journal of Ethnic and Migration Studies, 26(2), 229-245. <https://doi.org/10.1080/13691830050022785>

Bolzman, C. (1997). «Identidades colectivas, dinámica asociativa y participación social de las comunidades migrantes en Suiza». Revista Migraciones, 2, 75-98.

Cohen, J. y Arato, A. (2000). Sociedad Civil y Teoría Politica. México: Fondo de Cultura Económica.

Cortés, A. y SANMARTín, A. (2010). «Transnacionalismo político: Políticas migratorias de vinculación con los estados de origen y de las asociaciones migrantes en España. Los casos ecuatoriano y colombiano». XVI Encuentro de Latinoamericanos en España, 1.163.

Delgado, J. (2007). «Perspectivas clásicas y contemporáneas en el estudio de los movimientos sociales: Análisis multidimensional del giro hacia la relacionalidad». Revista Colombiana de Sociología, 28, 47-71.

Eisinger, P. (1973). «The Conditions of Protest Behavior in American Cities». American Political Science Review, 67(1), 11-28. $<$ https://doi.org/10.2307/1958525> 
Fennema, M. y Tillie, J. (2001). «Civic Community, Political Participation and Political Trust of Ethnic Groups». Connections, 24(1), 26-41. Recuperado de <https:// link.springer.com/chapter/10.1007/978-3-322-85129-1_9>.

GADEA, E. y AlBERT, M. (2011). «Asociacionismo inmigrante y renegociación de las identificaciones culturales». Politica y Sociedad, 48(1), 9-25.

GAMSON, W. Y MEYER, D. (1999). "Marcos interpretativos de la oportunidad política». En: MCAdam, D.; MCCARThY, J. y ZaLD, M. (comp.). Movimientos sociales: perspectivas comparadas. Madrid: ISTMO

GARRETA, J. (2011). "Las asociaciones de inmigrantes africanos: Tipologías y roles». En: García, F.J. y Kressova, N. Actas del I Congreso Internacional sobre Migraciones en Andalucía. Granada: Instituto de Migraciones, 199.

GOnZÁleZ, A.; Morales, L. y JorbA, L. (2009). «Políticas de incorporación y gestión del asociacionismo de la población de origen inmigrante a nivel local». En: ZAPAta-Barrero, R. (coord.). Politicas y gobernabilidad de la inmigración en España. Barcelona: Ariel, 113-138.

GONAALONS, P. (2007). «Oportunidades de participación política de las organizaciones de inmigrantes en España». Comunicación en el V Congreso de Migraciones. Valencia.

IRELAND, P.R. (1994). The policy challenge of ethnic diversity: Immigrant politics in France and Switzerland. Cambridge, Massachusetts: Harvard University Press.

JacobS, D. y Tillie, J. (2004). «Introduction: Social Capital and Political Integration of Migrants». Journal of Ethnic and Migration Studies, 30(5) (mayo), 419-427. <https://doi.org/10.1080/13691830410001682016>

KoOpmans, R. (2004). «Migrant Mobilization and Political Opportunities: Variation Among German Cities and a Comparison with the United Kingdom and the Netherlands». Journal of Ethnic and Migration Studies, 30(3), 449-470. <https://doi.org/10.1080/13691830410001682034>

Lacomba, J.; Boni, A.; Cloquell, A. y Soledad, C. (2014). «Inmigrants Associations and Co-Development Policies: Among the Opportunities for Strengthening and the Risk of Cooptation in the Case of Valencia Region (Spain). Voluntas (septiembre), 1-12. Recuperado de <https://link.springer.com/article/10.1007/ s11266-014-9491-9>.

MARKARIAN, V. (2006). Idos y recién llegados: La izquierda uruguaya en el exilio y las redes transnacionales de derechos humanos 1967-1984. Montevideo: Ediciones la Vasija, CIEU, Universidad de la República.

Martín Pérez, A. (2004). "Associations d'immigrés et politiques publiques en Espagne: Revendications, prestation de services et participation politique limitée». Migrations Société, 16(95), 15-28.

MATA, A. (2011). «Asociacionismo inmigrante y Administración local: Relaciones interdependientes». III Congreso Anual de la REPS.

McAdam, D. (1982). Political Process and the Development of Black Insurgency, 19301970. Chicago: University of Chicago Press.

McAdam, D.; McCarthy y MAYer, N. (1999). Movimientos sociales: Perspectivas comparadas. Madrid: Akal.

McAdam, D.; Tarrow, S. y Tilly, C. (2001). Dynamics of Contention. Cambridge: Cambridge University Press.

Melucci, A. (2002). Acción colectiva, vida cotidiana y democracia. México DF: Colegio de México.

Moncusí, A. y Escala, L. (2013). «Proyecciones transnacionales del asociacionismo migrante: Los ecuatorianos en Valencia, España». En: Esteban, Fernando O. 
(coord.). Espacios transnacionales de la migración latinoamericana en Europa. Buenos Aires: Antropofagia, 59-82.

Moraes, N. (2010). Transnacionalismo politico y nación: El papel del Estado y la sociedad civil migrante en la construcción de la trans-nación uruguaya. Universidad de Granada. Tesis doctoral.

Moraes, N.; BermúdeZ, A. y EscrivÁ, Á. (2013). "Las asociaciones de migrantes latinoamericanos como actores políticos en espacios locales y transnacionales». En: RAYA, E.; EsPADAS, M.A. y ABOUSSI, M. (eds.). Inmigración y ciudadanía activa. Barcelona: Icaria, 163-180.

Moraes, N.; Bermúdez, A.; Escrivá Á. y Padilla, B. (2009). «Estrategias de vinculación de los Estados Latinoamericanos con sus diásporas: Un análisis de las iniciativas desarrolladas por Colombia, Perú, Brasil y Uruguay». En: EsCRIVÁ, A.; Bermúdez, A. y Moraes, N. (eds.). Migración y participación politica: Estados, organizaciones y migrantes latinoamericanos en perspectiva local-transnacional. Madrid: CSIC, 297-326.

MorAlES, L. y GONZÁLEZ, L. (2006). «Las asociaciones de inmigrantes en Madrid: Una nota de investigación sobre su grado de integración política». Revista Española del Tercer Sector, 4 (septiembre-diciembre).

Morales, L.; GonZÁlez, A. y SÁnCheZ, G. (2005). «La integración política de los inmigrantes: Un estudio sobre las asociaciones de inmigrantes en Madrid y Murcia». Cuadernos Electrónicos de Filosofía del Derecho, 12.

Morales, L. y Mota, F. (2006). «El asociacionismo en España». En: MonTERO, J.R.; FONT, J. y TORCAL, M. (eds.). Ciudadanos, asociaciones y participación en España. Madrid: CIS.

MoYA, J. (2005). «Immigrants and associations: A global and historical perspective». Journal of Ethnic and Migration Studies, 31(5), 833-864. <https://doi.org/10.1080/13691830500178147>

Navarro, C.; Clark, T.; Delamata, G.; Herrera, R. y Pírez, P. (2006). Cambio social, estructura de oportunidades politicas y desarrollo cívico: Memoria proyecto de investigación. Centro de Estudios.

ØSTERGAARD-NIELSEN, E. (2003). «The politics of migrants» transnational political practices». International Migration Review, 37(3), 760-786. <https://doi.org/10.1111/j.1747-7379.2003.tb00157.x>

ØSTERGAARD-NiELSEN, E. (2009). «La política a través de las fronteras: Reflexiones sobre la dimensión transnacional de la participación política de los migrantes». En: EscrivÁ, A.; BermúdeZ, A. y Moraes, N. (eds.). Migración y participación politica: Estados, organizaciones y migrantes latinoamericanos en perspectiva localtransnacional. Madrid: CSIC.

Pedreño, A. (2005). «Sociedades etnofragmentadas». En: Pedreño, A. y Hernández, M. (coords.). La condición inmigrante. Exploraciones e investigaciones desde la Región de Murcia. Murcia: Universidad de Murcia, 75-106.

PERÒ, D. (2009). «Las movilizaciones políticas de los latinoamericanos en Londres». En: Escrivá, A.; Bermúdez, A. y Moraes, N. (edits.). Migración y participación politica: Estados, organizaciones y migrantes latinoamericanos en perspectiva localtransnacional. Madrid: CSIC, 155-182.

Però, D. y Solomos, J. (2010). «Introduction: Migrant Politics and Mobilization: Exclusion, Engagements, Incorporation». Ethnic and Racial Studies, 33(1), 1-18.

<https://doi.org/10.1080/01419870903418944> 
Portes, A.; Escobar, C. y Walton, A. (2006). «Organizaciones transnacionales de migrantes y desarrollo: Un estudio comparativo». Revista Migración y Desarrollo, 6 (primer semestre), 3-44.

Quiroga, M. (2009). "Movilización Social en Bolivia. La consolidación de lo indio como capital político». Revista Conflicto Social, 2(1), 168-194.

Quiroga, M. (2013). «Perspectivas para el análisis de la acción colectiva: Algunas reflexiones críticas y posibles aportes desde la teoría de la hegemonía». Araucaria: Revista Iberoamericana de Filosofía, Política y Humanidades, 15(30), 25-44.

Rucht, D. (1996). "The Impact of National Context on Social Movement Structures: A Cross-Movement and a cross-national comparison». En: MCADAM, D.; McCarthy, J. y Zald, M. (eds.). Comparative Perspectives on Social Movements: Political Opportunities, Mobilizing Structures and Cultural Framings. Cambridge: Cambridge University Press.

SChrover, M. y Vermeulen, F. (2005). «Immigrant Organizations». Journal of Ethnic and Migration Studies, 31(5), 823-832. <https://doi.org/10.1080/13691830500177792>

SCOTT, J.C. (2003). Los dominados y el arte de la resistencia. México: Txalapata.

Soysal, Y. (1994). Limits of Citizenship: Migrants and Postnational Membership in Europe. Chicago: University of Chicago Press.

STAtham, P. (1999). "Political mobilization by minorities in Britain: Negative feedback of "race relations"?». Journal of Ethnic and Migration Studies, 25(4), 597-626.

TARROW, S. (1997). El poder en movimiento: Los movimientos sociales, la acción colectiva y la politica. Madrid: Alianza Editorial.

Tilly, C. (1978). From Mobilization to Revolution. Nueva York: Random HouseMcGraw-Hill Publishing.

TORAL, M. (2010). «Las asociaciones de inmigrantes como sociedad civil: Un análisis tridimensional». Revista Española de Investigaciones Sociológicas, 132.

VEREDAS, S. (2003). «Las asociaciones de inmigrantes como sociedad civil: Un análisis tridimensional». Revista Española de Investigaciones Sociológicas, 36, 220.

Vermeulen, F. (2005). «Organisational patterns: Surinamese and Turkish associations in Amsterdam, 1960-1990». Journal of Ethnic and Migration Studies, 31(5), 951-973.

<https://doi.org/10.1080/13691830500177859> 